\title{
Power Variation and Time Change
}

\author{
Ole E. BARNDORFF-NIELSEN \\ Department of Mathematical Sciences, \\ University of Aarhus, Ny Munkegade, DK-8000 Aarhus C, Denmark \\ oebn@imf .au.dk \\ NEIL SHEPHARD \\ Nuffield College, University of Oxford, Oxford OX1 1NF, UK \\ neil.shephard@nuf.ox.ac.uk
}

August 16, 2004

\begin{abstract}
This paper provides limit distribution results for power variation, that is sums of powers of absolute increments, under nonequidistant subdivisions of time and for certain types of time-changed Brownian motion and $\alpha$-stable processes. Special cases of these processes are stochastic volatility models used extensively in financial econometrics.

Keywords: Power variation; r-variation; Realised variance; Semimartingales; Stochastic volatility; Time-change.
\end{abstract}

\section{Contents}

1 Introduction . . . . . . . . . . . . . . . . . . . . . . . . 1

2 Notation for power variation . . . . . . . . . . . . . . . . . . . . 3

3 Some Central Limit Theory results . . . . . . . . . . . . . . . . . . . . . . . . . . . . 4

3.1 Asymptotic normality ............................ 4

3.2 Probability limit results . . . . . . . . . . . . . . . . . . . . . . 4

4 Power variation under general subdivisions . . . . . . . . . . . . . . . . . . . 6

5 Power variation and time changed Brownian motion . . . . . . . . . . . . . . . 8

6 Power variation and time-changed stable processes . . . . . . . . . . . . . . . . . 11

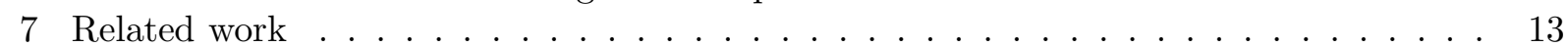

\section{Introduction}

In Barndorff-Nielsen and Shephard (2003) limit distribution results were derived for quantities of the form

$$
\left[X_{\delta}\right]^{[r]}(t)=\sum_{j=1}^{n}|X(j \delta)-X((j-1) \delta)|^{r},
$$

where $X$ denotes a special semimartingale with canonical decomposition of the form

$$
X=A+H \bullet W
$$

$r$ is a positive number and $n \delta=t$ for some time $t>0$. The theory is based on $\delta \downarrow 0$. We refer to (1.1) and similar quantities as power variations.

The initial limit results have been very substantially generalised and ramified in a number of recent publications. From the applied point of view the results in question provide, in particular, 
a versatile basis for drawing inference on the process $H$, which expresses the volatility of $X$, a key concept in the field of financial econometrics, e.g. the review in Andersen, Bollerslev, and Diebold (2004). This is discussed in Barndorff-Nielsen and Shephard (2002), Barndorff-Nielsen and Shephard (2003) and Barndorff-Nielsen and Shephard (2004). See also Shiryaev (1999, p. 349-350) who mentions interest in the limits of sums of absolute returns.

As a key illustration, suppose that $X$ is a Brownian semimartingale of the form

$$
X_{t}=\int_{0}^{t} a_{u} \mathrm{~d} u+\int_{0}^{t} H_{s_{-}} \mathrm{d} W
$$

where $a$ is predictable and locally bounded and $H$ is càdlàg. Then, under a very mild condition on $H$ (for the most general setting see Barndorff-Nielsen, Graversen, Jacod, Podolsky, and Shephard (2004)), for any $t>0$ and $\delta \downarrow 0$, we have

$$
\delta^{1-r / 2}\left[X_{\delta}\right]^{[r]}(t) \stackrel{p}{\rightarrow} \mu_{r} H^{r *}(t)
$$

where $\mu_{r}=\mathrm{E}\left\{|u|^{r}\right\}$ and $u \sim N(0,1)$ and

$$
H^{r *}(t)=\int_{0}^{t} H^{r}(s) \mathrm{d} s .
$$

Furthermore,

$$
\frac{\delta^{1-r / 2}\left[X_{\delta}\right]^{[r]}(t)-\mu_{r} H^{r *}(t)}{\delta^{1-r / 2} \sqrt{\mu_{2 r}^{-1} v_{r}\left[X_{\delta}\right]^{[2 r]}(t)}} \stackrel{\operatorname{law}}{\longrightarrow} N(0,1)
$$

where $v_{r}=\operatorname{Var}\left\{|u|^{r}\right\}$ is the variance of $|u|^{r}$.

Thus, in particular, we have that

$$
\frac{\left[X_{\delta}\right]^{[2]}(t)-H^{2 *}(t)}{\sqrt{\frac{2}{3}\left[X_{\delta}\right]^{[4]}(t)}} \stackrel{\text { law }}{\rightarrow} N(0,1)
$$

and

$$
\frac{\delta^{1 / 2}\left[X_{\delta}\right]^{[1]}(t)-\sqrt{2 / \pi} H^{*}(t)}{\sqrt{(1-2 / \pi) \delta\left[X_{\delta}\right]^{[2]}(t)}} \stackrel{\text { law }}{\rightarrow} N(0,1) .
$$

Result (1.4) appeared first in Barndorff-Nielsen and Shephard (2002), later work on this includes Mykland and Zhang (2005). See also the related Jacod (1994) and Jacod and Protter (1998). Result (1.5) is connected to some early work by Jacod (1994), but appeared in this form first in Barndorff-Nielsen and Shephard (2003).

The present paper indicates how these results may be extended to non-equidistant subdivisions of the time interval $[0, t]$ and it also considers more general time changes than those implicit in (1.2) (via the Dambis-Dubins-Schwarz theorem). Furthermore, settings where instead of the Brownian motion $W$ in (1.2) we have a symmetric $\alpha$-stable process will be discussed.

The structure of the paper is as follows. Section 2 introduces some notation and concepts for power variation, while in Section 3 we establish several consequences of the general central limit theory, needed for the core part of the paper. In Sections 4-6, we derive new limit law results for power variation in the case of nonequidistant time divisions and for time changed Brownian motion and symmetric $\alpha$-stable processes. (For simplicity, in these Sections, when discussing processes $X=A+H \bullet S$, where $S$ is either Brownian motion or symmetric stable, we assume that $A=0$ and that $H$ and $S$ are independent.) The final Section 7 mentions some related work. 


\section{Notation for power variation}

Let $\Delta$ denote a subdivision $0=t_{0}<t_{1}<\cdots<t_{n}=t$ of $[0, t]$ and let $\delta_{j}=t_{j}-t_{j-1}$ and $|\Delta|=\max \delta_{j}$. When considering a sequence of such subdivisions $\Delta$ we say that the sequence is balanced if $\max \delta_{j} / \min \delta_{j}$ is bounded above and $\varepsilon$-balanced, $\varepsilon \in(0,1)$, if $\max \delta_{j} /\left(\min \delta_{j}\right)^{\varepsilon} \rightarrow 0$ as $|\Delta| \rightarrow 0$. Clearly, if $\Delta$ is balanced then it is a fortiori $\varepsilon$-balanced for every $\varepsilon \in(0,1)$. Note that here and in the following we usually have in mind a single, generally unspecified, sequence of subdivisions $\Delta$ with $|\Delta| \rightarrow 0$; however, for notational simplicity, we do not indicate this by attaching a sequence index to $\Delta$.

We consider arbitrary real functions $f$ on the interval $[0, t]$ and introduce the notation

$$
\left[f_{\Delta}\right]^{[r]}=\sum\left|f\left(t_{j}\right)-f\left(t_{j-1}\right)\right|^{r}
$$

where the sum is over $j=1, \ldots, n$ and $r>0$. We call $\left[f_{\Delta}\right]^{[r]}$ the $r$-th order power variation of $f$ relative to $\Delta$, or the $r$-tic variation for short.

In the special case where the subdivision $\Delta$ is equidistant, whence $\delta_{j}=\delta$ for all $j$, we will write $f_{\delta}$ instead of $f_{\Delta}$, etc. Thus when $\delta$ occurs as an index the subdivision is understood to be equidistant. Furthermore, we write $[f]^{[r]}$ for the $r$-th order sup-variation or sup-r-variation ${ }^{1}$ of $f$, that is

$$
[f]^{[r]}=\sup _{\Delta \in \mathcal{D}} \sum\left|f\left(t_{j}\right)-f\left(t_{j-1}\right)\right|^{r}
$$

where $\mathcal{D}$ denotes the class of all possible subdivisions of $[0, t]$. When we wish to indicate the dependence on $t$ we shall write $\left[f_{\Delta}\right]^{[r]}(t)$ instead of $\left[f_{\Delta}\right]^{[r]}$, etc.

We define a time-change to be a non-decreasing function $T:[0, \infty) \rightarrow[0, \infty)$ with $T(0)=0$ and $T(t) \rightarrow \infty$ as $t \rightarrow \infty$.

For an arbitrary function $f$ (as above) and time-change $T$ we have

$$
\left[(f \circ T)_{\Delta}\right]^{[r]}=\left[f_{T(\Delta)}\right]^{[r]} \circ T
$$

(where o means composition of mappings) or, more specifically,

$$
\left[(f \circ T)_{\Delta}\right]^{[r]}(t)=\left[f_{T(\Delta)}\right]^{[r]}(T(t))
$$

where $T(\Delta)$ is the subdivision $0=T\left(t_{1}\right)<\cdots<T\left(t_{n}\right)=T(t)$.

Henceforth, unless otherwise mentioned, we assume that $T$ is continuous and strictly increasing. Then $T$ is uniformly continuous on any compact interval and $|\Delta| \rightarrow 0$ will imply $|T(\Delta)| \rightarrow 0$. Hence, in wide generality it will hold that

$$
[(f \circ T)]^{[r]}=[f]^{[r]} \circ T .
$$

Finally, when $f \geq 0$, we use the notation

$$
f^{*}(t)=\int_{0}^{t} f(s) \mathrm{d} s
$$

and, more generally,

$$
f^{r *}(t)=\int_{0}^{t} f^{r}(s) \mathrm{d} s .
$$

\footnotetext{
${ }^{1}$ We adopt this term rather than the more usual $r$-variation, for clarity in the context of the present paper. We will refer to some of the literature on $r$-variation in Section 7 of this paper.
} 


\section{Some Central Limit Theory results}

We shall need the following special cases of the general central limit theory.

Let $y_{n 1}, \ldots, y_{n k_{n}}\left(n=1,2, \ldots\right.$, with $k_{n} \rightarrow \infty$ as $\left.n \rightarrow \infty\right)$ be a triangular array of independent random variables and let $y_{n}=y_{n 1}+\cdots+y_{n k_{n}}$.

\subsection{Asymptotic normality}

Theorem 3.1 (Gnedenko and Kolmogorov (1954, p. 102-103)) Suppose that $\mathrm{E}\left\{y_{n j}\right\}=0$ for all $n$ and $j$ and that $\operatorname{Var}\left\{y_{n}\right\}=1$ for all $n$. Then $y_{n} \stackrel{\text { law }}{\longrightarrow} N(0,1)$ if and only if for arbitrary $\gamma>0$

$$
\sum_{j=1}^{k_{n}} \mathrm{E}\left\{y_{n j}^{2} \mathbf{1}_{(\gamma, \infty)}\left(\left|y_{n j}\right|\right)\right\} \rightarrow 0 .
$$

Corollary 3.1 Suppose that $y_{n j}$ is of the form $y_{n j}=c_{n j} x_{n j}$ where the $c_{n j}$ are real constants and the $x_{n j}$ are independent copies of a random variable $x$ that has mean 0 and variance 1. If $c_{n 1}^{2}+\cdots+c_{n k_{n}}^{2}=1$ and $c_{n}=\max _{j} c_{n j} \rightarrow 0$ as $n \rightarrow \infty$ then $y_{n}$ converges in law to the standard normal distribution $N(0,1)$.

PROOF In the present case

$$
\begin{aligned}
\sum_{j=1}^{k_{n}} \mathrm{E}\left\{y_{n j}^{2} \mathbf{1}_{(\gamma, \infty)}\left(\left|y_{n j}\right|\right)\right\} & =\sum_{j=1}^{k_{n}} c_{n j}^{2} \mathrm{E}\left\{x^{2} \mathbf{1}_{(\gamma, \infty)}\left(\left|c_{n j} x\right|\right)\right\} \\
& \leq \mathrm{E}\left\{x^{2} \mathbf{1}_{\left(c_{n}^{-1} \gamma, \infty\right)}(|x|)\right\} \rightarrow 0
\end{aligned}
$$

and hence Theorem 3.1 applies.

\subsection{Probability limit results}

Theorem 3.2 Degenerate Convergence Criterion (Loève (1977, p. 329)) We have that $y_{n} \stackrel{p}{\rightarrow} 0$ and the uniform asymptotic neglibility condition is satisfied if and only if for every $\varepsilon>0$ and for some $\gamma>0$

$$
\begin{gathered}
\sum_{j=1}^{k_{n}} P\left\{\left|y_{n j}\right| \geq \varepsilon\right\} \rightarrow 0 \\
\sum_{j=1}^{k_{n}} \mathrm{E}\left\{y_{n j} \mathbf{1}_{(-\gamma, \gamma)}\left(y_{n j}\right)\right\} \rightarrow 0
\end{gathered}
$$

and

$$
\left.\sum_{j=1}^{k_{n}}\left(\mathrm{E}\left\{y_{n j}^{2} \mathbf{1}_{(-\gamma, \gamma)}\right)\left(y_{n j}\right)\right\}-\mathrm{E}\left\{y_{n j} \mathbf{1}_{(-\gamma, \gamma)}\left(y_{n j}\right)\right\}^{2}\right) \rightarrow 0
$$

for $n \rightarrow \infty$.

Now, let $x_{n j}, n=1,2, \ldots, j=1,2, \ldots, k_{n}$ be independent copies of a random variable $x$ having distribution function $F$ and mean 0 , suppose that $c_{n i}$ are arbitrary positive reals and let

$$
y_{n j}=c_{n j} x_{n j}
$$

and $y_{n}=y_{n 1}+\cdots+y_{n k_{n}}$. 
Corollary 3.2 Suppose that $x$ has mean 0 , let $c_{n}=\max _{j} c_{n j}$ and assume that, as $n \rightarrow \infty$,

$$
\begin{gathered}
c_{n} \rightarrow 0 \\
k_{n} P\left\{|x| \geq c_{n}^{-1} \varepsilon\right\} \rightarrow 0 \\
\sup _{n} \sum_{j=1}^{k_{n}} c_{n j}<\infty
\end{gathered}
$$

and, for some $\gamma>0$,

$$
c_{n} \int_{-c_{n}^{-1} \gamma}^{c_{n}^{-1} \gamma} \xi^{2} \mathrm{~d} F(\xi) \rightarrow 0 .
$$

Then $y_{n} \stackrel{p}{\rightarrow} 0$.

Proof In the present setting the conditions of Theorem 3.2 take the form

$$
\begin{gathered}
\sum_{j=1}^{k_{n}} P\left\{|x| \geq c_{n j}^{-1} \varepsilon\right\} \rightarrow 0 \\
\sum_{j=1}^{k_{n}} c_{n j} \int_{-c_{n j}^{-1} \gamma}^{c_{n j}^{-1} \gamma} \xi \mathrm{d} F(\xi) \rightarrow 0
\end{gathered}
$$

and

$$
\sum_{j=1}^{k_{n}} c_{n j}^{2}\left(\int_{-c_{n j}^{-1} \gamma}^{c_{n j}^{-1} \gamma} \xi^{2} \mathrm{~d} F(\xi)-\left(\int_{-c_{n j}^{-1} \gamma}^{c_{n j}^{-1} \gamma} \xi \mathrm{d} F(\xi)\right)^{2}\right) \rightarrow 0 .
$$

The first of these conditions is implied by (3.5) and (3.6). Next, since $\mathrm{E}\{x\}=0$ and $c_{n} \rightarrow 0$,

$$
\int_{-c_{n j}^{-1} \gamma}^{c_{n j}^{-1} \gamma} \xi \mathrm{d} F(\xi) \rightarrow 0
$$

uniformly in $j$. Combined with (3.7) the latter entails (3.10) and also

$$
\sum_{j=1}^{k_{n}} c_{n j}^{2}\left(\int_{-c_{n j}^{-1} \gamma}^{c_{n j}^{-1} \gamma} \xi \mathrm{d} F(\xi)\right)^{2} \rightarrow 0 .
$$

Finally (3.8) gives

$$
\sum_{j=1}^{k_{n}} c_{n j}^{2} \int_{-c_{n j}^{-1} \gamma}^{c_{n j}^{-1} \gamma} \xi^{2} \mathrm{~d} F(\xi) \leq c_{n} \int_{-c_{n}^{-1} \gamma}^{c_{n}^{-1} \gamma} \xi^{2} \mathrm{~d} F(\xi) \sum_{j=1}^{k_{n}} c_{n j} \rightarrow 0 .
$$

Corollary 3.3 Suppose that $x$ has mean 0 and finite variance and assume that

$$
\begin{gathered}
c_{n} \rightarrow 0 \\
k_{n} P\left\{|x| \geq c_{n}^{-1} \varepsilon\right\} \rightarrow 0 \\
\sup _{n} \sum_{j=1}^{k_{n}} c_{n j}<\infty .
\end{gathered}
$$

Then $y_{n} \stackrel{p}{\rightarrow} 0$.

Proof Condition (3.8) follows from the assumed finiteness of $\operatorname{Var}\{x\}$. 


\section{Power variation under general subdivisions}

We shall now derive a partial extension of (1.3) to cases of non-equidistant $\Delta$. Convergence statements will refer to a sequence of subdivisions $\Delta$ with $|\Delta| \rightarrow 0$.

For simplicity we assume that the process $a=0$, i.e. $X$ is of the form $X=H \bullet W$ with $H$ being càdlàg and independent of the Brownian motion $W$. We let $Q=H^{2}$.

It is now convenient to introduce the notation

$$
\left\{X_{\Delta}\right\}^{[r]}=\sum \delta_{j}^{1-r / 2}\left|X\left(t_{j}\right)-X\left(t_{j-1}\right)\right|^{r}
$$

and the condition

(V) The volatility process $H$ is (pathwise) bounded away from 0 and has, moreover, the property that for some $\gamma>0$ (equivalently for all $\gamma>0$ )

$$
\frac{\sum_{j=1}^{m} \delta_{j}\left|H^{\gamma}\left(\eta_{j}\right)-H^{\gamma}\left(\xi_{j}\right)\right|}{\sqrt{\min \delta_{j}}} \rightarrow 0
$$

for any sequences $\xi_{j}=\xi_{j}(\Delta)$ and $\eta_{j}=\eta_{j}(\Delta)$ satisfying

$$
0 \leq \xi_{1} \leq \eta_{1} \leq t_{1} \leq \xi_{2} \leq \eta_{2} \leq t_{2} \leq \cdots \leq \xi_{n} \leq \eta_{n} \leq t .
$$

Now recall the definition of an $\varepsilon$-balanced sequence of subdivisions $\Delta$, given in Section 2 .

Theorem 4.1 Let $X$ be a semimartingale of the form $X=H \bullet W$ and suppose that the volatility process $H$ is independent of the Brownian motion $W$ and satisfies condition (V). Then, for any $t>0$ and for any $\frac{1}{2}$-balanced sequence of subdivisions $\Delta$ we have

$$
\left\{X_{\Delta}\right\}^{[r]}(t) \stackrel{p}{\rightarrow} \mu_{r} H^{r *}(t)
$$

as $|\Delta| \rightarrow 0$ and where $\mu_{r}=\mathrm{E}\left\{|u|^{r}\right\}$ and $u \sim N(0,1)$.

Furthermore, if the sequence of subdivisions $\Delta$ is $\frac{2}{3}$-balanced then

$$
\frac{\left\{X_{\Delta}\right\}^{[r]}(t)-\mu_{r} H^{r *}(t)}{\sqrt{\mu_{2 r}^{-1} \nu_{r} \sum \delta_{j}^{2-r}\left|X\left(t_{j}\right)-X\left(t_{j-1}\right)\right|^{2 r}(t)}} \stackrel{\text { law }}{\rightarrow} N(0,1)
$$

where $\nu_{r}=\operatorname{Var}\left\{|u|^{r}\right\}$ is the variance of $|u|^{r}$.

Proof We have

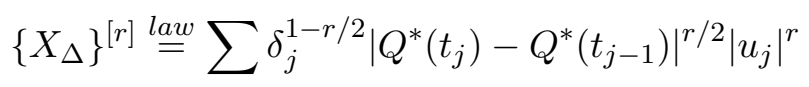

where the $u_{j}$ are i.i.d. standard normal. Hence, for arbitrary $\gamma>0$, letting

$$
\left\{Q_{\Delta}^{*}\right\}^{[\gamma]}=\sum \delta_{j}^{1-\gamma}\left|Q^{*}\left(t_{j}\right)-Q^{*}\left(t_{j-1}\right)\right|^{\gamma},
$$

we find

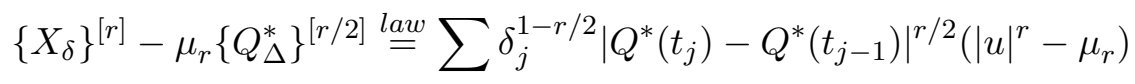

and it follows from Corollary 3.1 that

$$
\frac{\left.\left\{X_{\delta}\right\}^{[r]}(t)-\mu_{r}\left\{Q_{\Delta}^{*}\right\}^{[r / 2]} t\right)}{\sqrt{v_{r} \sum \delta_{j}^{2-r}\left|Q^{*}\left(t_{j}\right)-Q^{*}\left(t_{j-1}\right)\right|^{r}}} \stackrel{\text { law }}{\longrightarrow} N(0,1)
$$


provided

$$
\frac{\max \left\{\delta_{j}^{1-r / 2}\left|Q^{*}\left(t_{j}\right)-Q^{*}\left(t_{j-1}\right)\right|^{r / 2}\right\}}{\sqrt{\sum \delta_{j}^{2-r}\left|Q^{*}\left(t_{j}\right)-Q^{*}\left(t_{j-1}\right)\right|^{r}}} \rightarrow 0 .
$$

To show that the latter is the case we note that

$$
\begin{aligned}
\frac{\max \left\{\delta_{j}^{1-r / 2}\left|Q^{*}\left(t_{j}\right)-Q^{*}\left(t_{j-1}\right)\right|^{r / 2}\right\}}{\sqrt{\sum \delta_{j}^{2-r}\left|Q^{*}\left(t_{j}\right)-Q^{*}\left(t_{j-1}\right)\right|^{r}}} & \leq \frac{\max \delta_{j}}{\sqrt{\min \delta_{j}}} \frac{\max \left\{\delta_{j}^{-r / 2}\left|Q^{*}\left(t_{j}\right)-Q^{*}\left(t_{j-1}\right)\right|^{r / 2}\right\}}{\sqrt{\sum \delta_{j}^{-r}\left|Q^{*}\left(t_{j}\right)-Q^{*}\left(t_{j-1}\right)\right|^{r}}} \\
& =\frac{\max \delta_{j}}{\sqrt{\min \delta_{j}}} \frac{\max \phi_{j}^{r / 2}}{\sqrt{\sum \delta_{j} \phi_{j}^{r}}}
\end{aligned}
$$

where $\phi_{j}$ is given by

$$
\delta_{j}^{-1}\left|Q^{*}\left(t_{j}\right)-Q^{*}\left(t_{j-1}\right)\right|=\phi_{j} .
$$

By the càdlàg property of $H$ we have $\phi_{j} \leq \sup _{0 \leq s \leq t} Q(s)<\infty$ and, for any $\gamma>0$,

$$
\sum \delta_{j} \phi_{j}^{\gamma} \rightarrow \int_{0}^{t} Q^{\gamma}(s) \mathrm{d} s=Q^{\gamma *}(t)
$$

which, together with (4.7) and the assumption that the sequence of subdivisions $\Delta$ is $\frac{1}{2}$-balanced implies that (4.6) is fulfilled. Hence (4.5) has been shown to hold.

By (4.9) we also have

$$
\left\{Q_{\Delta}^{*}\right\}^{[\gamma]} \rightarrow Q^{\gamma *}=H^{2 \gamma *}
$$

for every $\gamma>0$ and therefore, in view of (4.5), we will have

$$
\frac{\left\{X_{\delta}\right\}^{[r]}(t)-\mu_{r} H^{r *}(t)}{\sqrt{v_{r} \sum \delta_{j}^{2-r}\left|Q^{*}\left(t_{j}\right)-Q^{*}\left(t_{j-1}\right)\right|^{r}}} \stackrel{\text { law }}{\rightarrow} N(0,1)
$$

provided

$$
\frac{\left\{Q_{\Delta}^{*}\right\}^{[r / 2]}(t)-Q^{r / 2 *}(t)}{\sqrt{\sum \delta_{j}^{2-r} \mid Q^{*}\left(t_{j}\right)-Q^{*}\left(t_{j-1}\right)^{r}}} \rightarrow 0 .
$$

The numerator in (4.12) may be rewritten as

$$
\begin{aligned}
\left\{Q_{\Delta}^{*}\right\}^{[r / 2]}(t)-Q^{r / 2 *}(t) & =\sum \delta_{j}\left(\delta_{j}^{-r / 2}\left|Q^{*}\left(t_{j}\right)-Q^{*}\left(t_{j-1}\right)\right|^{r / 2}-\delta_{j}^{-1} \int_{t_{j-1}}^{t_{j}} Q^{r / 2}(s) \mathrm{d} s\right) \\
& =\sum \delta_{j}\left(\phi_{j}^{r / 2}-\psi_{j}^{r / 2}\right)
\end{aligned}
$$

where $\phi_{j}$ was defined by (4.8) and

$$
\psi_{j}=\left(\delta_{j}^{-1} \int_{t_{j-1}}^{t_{j}} Q^{r / 2}(s) \mathrm{d} s\right)^{2 / r} .
$$

(For simplicity, we have suppressed the dependence of $\psi_{j}$ on $r$ in the notation.) For the denominator we have

$$
\min \delta_{j}\left\{Q_{\Delta}^{*}\right\}^{[r]}(t) \leq \sum \delta_{j}^{2-r}\left|Q^{*}\left(t_{j}\right)-Q^{*}\left(t_{j-1}\right)\right|^{r} \geq \max \delta_{j}\left\{Q_{\Delta}^{*}\right\}^{[r]}(t) .
$$


Thus

$$
\frac{\left\{Q_{\Delta}^{*}\right\}^{[r / 2]}(t)-Q^{r / 2 *}(t)}{\sqrt{\sum \delta_{j}^{2-r}\left|Q^{*}\left(t_{j}\right)-Q^{*}\left(t_{j-1}\right)\right|^{r}}} \leq \frac{1}{\sqrt{\left\{Q_{\Delta}^{*}\right\}^{[r]}(t)}} \frac{\sum \delta_{j}\left(\phi_{j}^{r / 2}-\psi_{j}^{r / 2}\right)}{\sqrt{\min \delta_{j}}}
$$

and on account of (4.10) and condition ( $\mathbf{V})$ the right hand side tends to 0, verifying (4.12) and hence (4.11).

Since by (4.14) and (4.9) the denominator in (4.11) tends to 0 we have shown the first assertion in Theorem 4.1 .

It remains to prove that, under the strengthened assumption that the sequence of subdivisions is $\frac{2}{3}$-balanced, we may substitute

$$
\mu_{2 r}^{-1} \sum \delta_{j}^{2-r}\left|X\left(t_{j}\right)-X\left(t_{j-1}\right)\right|^{r}(t)
$$

for

$$
\sum \delta_{j}^{2-r}\left|Q^{*}\left(t_{j}\right)-Q^{*}\left(t_{j-1}\right)\right|^{r}
$$

in (4.11). Noting that

$$
\sum \delta_{j}^{2-r}\left|Q^{*}\left(t_{j}\right)-Q^{*}\left(t_{j-1}\right)\right|^{r}=\sum \delta_{j}^{2} \phi_{j}^{r}
$$

and

$$
\sum \delta_{j}^{2-r}\left|X\left(t_{j}\right)-X\left(t_{j-1}\right)\right|^{r}-\mu_{2 r} \sum \delta_{j}^{2-r}\left|Q^{*}\left(t_{j}\right)-Q^{*}\left(t_{j-1}\right)\right|^{r} \stackrel{l a w}{=} \sum \delta_{j}^{2} \phi_{j}^{r}\left(\left|u_{j}\right|^{2 r}-\mu_{2 r}\right)
$$

we must, in other words, prove that $\sum \delta_{j}^{2} \phi_{j}^{r}\left(\left|u_{j}\right|^{2 r}-\mu_{2 r}\right)$ is of smaller order of magnitude than $\sum \delta_{j}^{2} \phi_{j}^{r}\left|u_{j}\right|^{2 r}$, in probability as $|\Delta| \rightarrow 0$. For this it is enough to show that the standard deviation of the former sum is of smaller order than the mean of the latter sum. The ratio of these two quantities is

$$
\frac{\sqrt{\nu_{2 r}}}{\mu_{2 r}} \frac{\sqrt{\sum \delta_{j}^{4} \phi_{j}^{2 r}}}{\sum \delta_{j}^{2} \phi_{j}^{r}}
$$

where for the second ratio we have

$$
\frac{\sqrt{\sum \delta_{j}^{4} \phi_{j}^{2 r}}}{\sum \delta_{j}^{2} \phi_{j}^{r}} \leq \frac{\left(\max \delta_{j}\right)^{3 / 2}}{\min \delta_{j}} \frac{\sqrt{\sum \delta_{j} \phi_{j}^{2 r}}}{\sum \delta_{j} \phi_{j}^{r}}
$$

The second ratio on the right hand side of this inequality is bounded, by a previous argument, and the first ratio tends to 0 on account of the $\frac{2}{3}$-balancedness assumption.

Example 4.1 If the sequence of subdivisions $\Delta$ is balanced and if $H$ is of local bounded variation then condition (4.2) is satisfied. The latter requirement is met in particular by the superpositions of OU processes used as models for $H$ in Barndorff-Nielsen and Shephard (2001b), Barndorff-Nielsen and Shephard (2001a), cf. also Barndorff-Nielsen (2001) and BarndorffNielsen, Nicolato, and Shephard (2002).

\section{Power variation and time changed Brownian motion}

Our focus in this Section is on time changed Brownian motion, that is we are considering local martingales of the form

$$
X=B \circ T
$$


and we aim to extend (1.3) to this setting, moreover allowing the subdivisions $\Delta$ to be nonequidistant.

Processes of the form $X=H \bullet W$, where $H$ is càdlàg, fall within this group. In fact, supposing that

$$
H^{2 *}(t)=\int_{0}^{t} H^{2}(s) \mathrm{d} s \rightarrow \infty
$$

for $t \rightarrow \infty$ we have, by the Dambis-Dubins Schwarz theorem ${ }^{2}$, that the process $X=H \bullet W$ can be reexpressed a.s. as $B \circ T$ where $T=H^{2 *}=Q^{*}$ and the Brownian motion $B$ is defined from $X$ by $B=X \circ \overleftarrow{T}$ where $\overleftarrow{T}$ denotes the inverse of the time change of $T$. (Of course, $T$ and $\overleftarrow{T}$ are themselves determined by $X$ since $T=H^{2 *}=[X]$, the quadratic variation of $X$.)

We assume that the time-change $T$ is continuous and strictly increasing. As before, $\Delta$ stands for a subdivision $0=t_{0}<t_{1}<\cdots<t_{n}=t$ of $[0, t]$ (with $t$ and $n$ suppressed in some of the notation), and the limit statements refer to a single, but arbitrary, sequence of subdivisions $\Delta$ with $|\Delta| \rightarrow 0$. Further, we assume that $T$ is independent of $B$, and therefore we may argue conditionally on $T$. Otherwise put, we may consider $T$ to be deterministic.

Letting

$$
T_{\Delta j}=T\left(t_{j}\right)-T\left(t_{j-1}\right)
$$

we have

$$
\left[X_{\Delta}\right]^{[r]}-\mu_{r}\left[T_{\Delta}\right]^{[r / 2]} \stackrel{\text { law }}{=} \sum_{j=1}^{M} T_{\Delta j}^{r / 2}\left(\left|u_{j}\right|^{r}-\mu_{r}\right)
$$

where $u_{1}, \ldots, u_{n}$ are independent copies of a standard normal variate $u$ (and, as before, $\mu_{r}=$ $\left.\mathrm{E}\left\{|u|^{r}\right\}\right)$. Consequently,

$$
\frac{\left[X_{\Delta}\right]^{[r]}-\mu_{r}\left[T_{\Delta}\right]^{[r / 2]}}{\sqrt{v_{r}\left[T_{\Delta}\right]^{[r]}}} \stackrel{\text { law }}{=} y_{\Delta}
$$

where $y_{\Delta}=y_{\Delta 1}+\cdots+y_{\Delta n}$ and $y_{\Delta j}=c_{\Delta j} x_{\Delta j}$ with

$$
c_{\Delta j}=\frac{T_{\Delta j}^{r / 2}}{\sqrt{\left[T_{\Delta}\right]^{[r]}}}
$$

and $x_{\Delta j} \stackrel{\text { law }}{=}\left(|u|^{r}-\mu_{r}\right) / \sqrt{v_{r}}$. By Corollary 3.1 we obtain

Theorem 5.1 Suppose that

$$
\frac{\max _{j} T_{\Delta j}^{r / 2}}{\sqrt{\left[T_{\Delta}\right]^{[r]}}} \rightarrow 0
$$

as $|\Delta| \rightarrow 0$. Then

$$
\frac{\left[X_{\Delta}\right]^{[r]}-\mu_{r}\left[T_{\Delta}\right]^{[r / 2]}}{\sqrt{v_{r}\left[T_{\Delta}\right]^{[r]}}} \stackrel{\text { law }}{\rightarrow} N(0,1) .
$$

Example 5.1 Suppose $r=1$. Then $\left[T_{\Delta}\right]^{[r]}=T(t)$ and, since $T$ is uniformly continuous on $[0, t]$, condition (5.2) holds. More generally, since, by Jensen's inequality, $\left(\left[T_{\Delta}\right]^{[r]}\right)^{1 / r}$ is decreasing in $r$ we have for $r \leq 1$ that

$$
\sqrt{\left[T_{\Delta}\right]^{[r]}} \geq T(t)^{r / 2}
$$

\footnotetext{
${ }^{2}$ The extension of this theorem to the case where instead of the Brownian motions $W$ and $B$ one considers stable processes is discussed in the recent paper by Kallsen and Shiryaev (2002), the results of which are summarised in Section 6 below.
} 
and hence (5.2) is, in fact, valid for all $0<r \leq 1$.

Note that, writing $\hat{T}_{\Delta}=\max _{j} T_{\Delta j}$ we have

$$
\frac{\max _{j} T_{\Delta j}^{r / 2}}{\sqrt{\left[T_{\Delta}\right]^{[r]}}}=\left\{\sum\left(T_{\Delta j} / \hat{T}_{\Delta}\right)^{r}\right\}^{-1 / 2} .
$$

Example 5.2 Suppose $T(s)=s^{\psi}$ for some $\psi \in(0,1)$ and, for simplicity, take $t=1$. Taking $\Delta$ to be the equidistant subdivision determined by $t_{j}=j / n$ we have $\hat{T}_{\Delta}=n^{-\psi}$ and

$$
\sum\left(T_{\Delta j} / \hat{T}_{\Delta}\right)^{r}=\sum\left\{j^{\psi}-(j-1)^{\psi}\right\}^{r}
$$

where for large $j$

$$
\left(j^{\psi}-(j-1)^{\psi}\right)^{r} \sim \psi^{r} j^{-(1-\psi) r} .
$$

Consequently, if $(1-\psi) r>1$ condition (5.2) is not satisfied. In particular, this is the case if $r=2$ and $\psi<\frac{1}{2}$.

Example 5.3 In case $T=Q^{*}$, where

$$
Q^{*}(s)=\int_{0}^{s} Q(u) \mathrm{d} u
$$

for some positive Riemann integrable function $Q$ on $[0, t]$, we have

$$
\underline{Q} \leq \Delta_{j}^{-1} T_{\Delta j} \leq \bar{Q}
$$

where $Q$ and $\bar{Q}$ are, respectively, the infimum and the supremum of $Q$ over $[0, t]$. Suppose further that $Q$ is bounded away from 0 , i.e. $Q>0$.

Then we have

$$
\frac{\max _{j} T_{\Delta j}^{r / 2}}{\sqrt{\left[T_{\Delta}\right]^{[r]}}} \leq \frac{1}{\sqrt{n}}\left(\frac{\max \delta_{j}}{\min \delta_{j}}\right)^{r / 2}(\bar{Q} / \underline{Q}) /^{r / 2} \rightarrow 0
$$

and it follows that condition (5.2) is satisfied and Theorem 5.1 applies if $\max \delta_{j} / \min \delta_{j}$ is bounded above, as is the case in particular if the subdivision $\Delta$ is equidistant.

Now suppose that $\left[T_{\Delta}\right]^{[r / 2]}$ converges as $|\Delta| \rightarrow 0$, with limit $[T]^{[r / 2]}$, irrespectively of which sequence of subdivisions is considered. It is then of interest to consider conditions under which

$$
\frac{\left[X_{\Delta}\right]^{[r]}-\mu_{r}[T]^{[r / 2]}}{\sqrt{v_{r}\left[T_{\Delta}\right]^{[r]}}} \stackrel{\text { law }}{\rightarrow} N(0,1) .
$$

Clearly this will be the case provided

$$
\frac{\left[T_{\Delta}\right]^{[r / 2]}-[T]^{[r / 2]}}{\sqrt{\left[T_{\Delta}\right]^{[r]}}} \rightarrow 0
$$

as $|\Delta| \rightarrow 0$. In particular, for $r=2$ we have simply $\left[T_{\Delta}\right]^{[r / 2]}=[T]^{[r / 2]}$ and therefore the following Corollary to Theorem 5.1.

Corollary 5.1 If

$$
\frac{\max _{j} T_{\Delta j}}{\sqrt{\left[T_{\Delta}\right]^{[2]}}} \rightarrow 0
$$

as $|\Delta| \rightarrow 0$ then

$$
\frac{\left[X_{\Delta}\right]^{[2]}(t)-T(t)}{\sqrt{2\left[T_{\Delta}\right]^{[2]}}} \stackrel{\text { law }}{\rightarrow} N(0,1) .
$$




\section{Power variation and time-changed stable processes}

We now inquire into the question of the degree to which the results discussed above for timechanged Brownian motion can be extended to the class of $\alpha$-stable processes. For simplicity we restrict attention to the case where $X$ is of the form $X=H \bullet Z$ for some symmetric $\alpha$-stable Lévy process, and we consider only equidistant subdivisions.

We first recall some known facts about symmetric $\alpha$-stable processes. Let $Z$ be the symmetric $\alpha$-stable process with $0<\alpha<2$ and cumulant function

$$
\mathrm{C}\{\zeta \ddagger Z(t)\}=\log \mathrm{E} e^{i \zeta Z(t)}=-t|\zeta|^{\alpha} .
$$

This process is representable by subordination as

$$
Z(t) \stackrel{\operatorname{law}}{=} B(S(t))
$$

where $S$ is the positive $\alpha / 2$-stable subordinator with kumulant function

$$
\overline{\mathrm{K}}\{\theta \ddagger S(t)\}=\log \mathrm{E} e^{-\theta S(t)}=-t(2 \theta)^{\alpha / 2} .
$$

When $r<\alpha$, which is needed for the moments to exist, we will write

$$
\mu_{\alpha, r}=\mathrm{E}\left\{|Z(1)|^{r}\right\}=\mu_{r} \mathrm{E}\left\{S(1)^{r / 2}\right\} .
$$

Furthermore, if $H$ is a predictable process such that for all $t>0$

$$
\int_{0}^{t}|H|_{s}^{\alpha} \mathrm{d} s<\infty
$$

and, for $t \rightarrow \infty$,

$$
\int_{0}^{t}|H|_{s}^{\alpha} \mathrm{d} s \rightarrow \infty
$$

then

$$
H \bullet Z=\tilde{Z} \circ|H|^{\alpha *}
$$

where $\tilde{Z}$ is a symmetric $\alpha$-stable process and (in the previously established notation)

$$
|H|_{t}^{\alpha *}=\int_{0}^{t}|H|_{s}^{\alpha} \mathrm{d} s
$$

Remark In the case where $H$ is nonnegative the same conclusion holds for arbitrary, i.e. not necessarily symmetric, $\alpha$-stable processes. For a proof and the history of these result, see Kallsen and Shiryaev (2002). These authors also show that, in essence, the results cannot be extended to more general Lévy processes.

Henceforth, let $H(t)$ be a nonnegative and locally Riemann integrable function on $[0, \infty)$ and assume that

$$
\int_{0}^{t} H^{\alpha}(s) \mathrm{d} Z(s)<\infty
$$

for all $t>0$. Then $X=H \bullet Z$ is a well-defined process. In line with the previous discussion, we assume that $H$ and $Z$ are independent, and we write $X_{j}=X(j \delta)-X((j-1) \delta)$ and $Z_{j}=$ $Z(j \delta)-Z((j-1) \delta)$.

As an initial consideration we look at the asymptotic behaviour of unnormalised power variations and let $H \equiv 1$, i.e. we consider the simplest case, $X=Z$. Recall first that the 
sup-variation $[Z]^{[r]}(t)$ is finite or infinite according to whether $r>\alpha$ or $r \leq \alpha$ (cf. Fristedt and Taylor (1973), Mikosch and Norvaiša (2000)).

The law of $\left|X_{j}\right|$ is the same as the law of $\delta^{1 / \alpha}|Z(1)|$ and thus

$$
\left[X_{\delta}\right]^{[r]}(t) \stackrel{\text { law }}{=} \delta^{r / \alpha} \sum_{j=1}^{M}\left|Z_{j}\right|^{r} .
$$

The random variables $\left|Z_{j}\right|$ belong to the domain of normal attraction of a stable law with index $\alpha$. Hence, on account of Feller (1971, pp. 580-581), we have the following limit properties, where for simplicity we are letting $r=1$ :

If $1<\alpha<2$ then, for a certain $\alpha$-stable law $S_{\alpha}$,

$$
\left[X_{\delta}\right]^{[1]}(t)-\delta^{-1+1 / \alpha} \mu_{\alpha, 1} \stackrel{\operatorname{law}}{\rightarrow} S_{\alpha}
$$

If $0<\alpha<1$ then, for a certain positive $\alpha$-stable law $S_{+\alpha}$,

$$
\left[X_{\delta}\right]^{[1]}(t) \stackrel{\text { law }}{\longrightarrow} S_{+\alpha}
$$

If $\alpha=1$ then, for a certain 1-stable law $S_{1}$,

$$
\left[X_{\delta}\right]^{[1]}(t)-b_{\delta} \stackrel{\text { law }}{\rightarrow} S_{1}
$$

where

$$
b_{\delta}=\int_{-\infty}^{\infty} \sin (\delta x) \mathrm{d} P\{|Z(1)| \leq x\} .
$$

In all three cases, $\delta^{1 / 2}\left[X_{\delta}\right]^{[1]}(t) \stackrel{p}{\rightarrow} 0$. Note that the above limit laws are more complicated than the mixed Gaussian limit laws obtained in Sections 3 and 5.

Next, for general $H$ we have, by (6.1) and (6.2),

$$
X_{j} \stackrel{\operatorname{law}}{=}\left(\int_{(j-1) \delta}^{j \delta} H^{\alpha}(s) \mathrm{d} s\right)^{1 / \alpha} Z(1)
$$

so that

$$
\left|X_{j}\right|^{r} \stackrel{\operatorname{law}}{=}\left(\int_{(j-1) \delta}^{j \delta} H^{\alpha}(s) \mathrm{d} s\right)^{r / \alpha}\left|v_{j}\right|^{r}
$$

where $v_{1}, \ldots, v_{M}$ are i.i.d. with the same distribution as $Z(1)$. Equivalently, by the subordination property, we have

$$
\left|X_{j}\right|^{r} \stackrel{\operatorname{law}}{=}\left(\int_{(j-1) \delta}^{j \delta} H^{\alpha}(s) \mathrm{d} s\right)^{r / \alpha} q_{j}^{r / 2}\left|u_{j}\right|^{r}
$$

where the $q_{1}, \ldots, q_{M}$ are i.i.d., with the same law as $S(1)$ and are independent of $u_{1}, \ldots, u_{M}$ which are i.i.d. standard normal.

In view of these representations of $\left|X_{j}\right|^{r}$ it would be rather simple to give a complete description of the various possible limiting behaviours of realised power variation as $\delta \rightarrow 0$. Here we shall only discuss some particular cases. 
For $r=2$ we have that realised quadratic variation is

$$
\left[X_{\delta}\right](t) \stackrel{\operatorname{law}}{=}\left\{\sum_{j=1}^{n}\left|u_{j}\right|^{\alpha} \int_{(j-1) \delta}^{j \delta} H^{\alpha}(s) \mathrm{d} s\right\}^{2 / \alpha} S(1) .
$$

The term in braces satisfies, conditionally on $H$, as $\delta \downarrow 0$

$$
\sum_{j=1}^{M}\left|u_{j}\right|^{\alpha} \int_{(j-1) \delta}^{j \delta} H^{\alpha}(s) \mathrm{d} s \stackrel{p}{\rightarrow} \mu_{\alpha} H^{\alpha *}(t) .
$$

This follows from Corollary 3.3. Consequently, for the quadratic variation we have

$$
\left[X^{*}\right](t) \stackrel{\operatorname{law}}{=}\left\{\mu_{\alpha} H^{\alpha *}(t)\right\}^{2 / \alpha} S(1) .
$$

Much simpler and statistically more powerful results are available if we use realised power variation instead of realised quadratic variation.

Recall $\mathrm{E}|Z(1)|^{\gamma}$ exists if (and only if) $\gamma<\alpha$. Thus the moments of $|Z(1)|^{r}$ exist up to, but not including, order $\alpha / r$. Hence, still given $H$, if $r<\alpha$ and $1<\alpha<2$ then

$$
\delta^{1-r / \alpha}\left[X_{\delta}\right]^{[r]}(t) \stackrel{p}{\rightarrow} \mu_{\alpha, r} H^{r *}(t),
$$

(where $\mu_{\alpha, r}=\mathrm{E}\left\{|Z(1)|^{r}\right\}$ ). This may be verified by means of Corollary 3.2. In fact, the assumptions made on $H$ imply that it suffices to prove the statement in the case $H \equiv 1$. Then, in the notation of Corollary 3.2, $c_{n}=n^{-1}$ and the conditions (3.5)-(3.8) are easily checked using the well known tail behaviour of the $\alpha$-stable laws. (The result (6.5) provides a simple generalisation of the use of quadratic variation for Brownian motion based stochastic volatility models, for then $r=2$ and

$$
\left[X_{\delta}\right]^{[2]}(t) \stackrel{p}{\rightarrow} H^{2 *}(t)
$$

exactly.)

In case $r<\alpha / 2$ we have the stronger result that

$$
\frac{\delta^{1-r / \alpha} \mu_{\alpha, r}^{-1}\left[X_{\delta}\right]^{[r]}(t)-H^{r *}(t)}{\delta^{1 / 2} \sqrt{\mu_{\alpha, r}^{-2} v_{\alpha, r} H^{2 r *}(t)}} \stackrel{\text { law }}{\rightarrow} N(0,1),
$$

where $v_{\alpha, r}=\operatorname{Var}\left\{|z(1)|^{r}\right\}$. This result holds both conditionally and unconditionally. This is a consequence of Corollary 4.1.

Of course in practice the above limit theory is has an unknown denominator $H^{2 r *}(t)$ and so could not be used even if we were to know $\alpha$. However, in theory we could replace $H^{2 r *}(t)$ by the consistent estimator

$$
\delta^{1-2 r / \alpha} \mu_{\alpha, 2 r}^{-1}\left[X_{\delta}\right]^{[2 r]}(t)
$$

\section{Related work}

There are in the literature a considerable number of important results on power variations of semimartingales generally, and Lévy processes in particular, that are related but not directly relevant to what we have discussed above. To complete the picture the following points contain a brief guide to those results.

Power variation and Lévy processes A number of authors have investigated the relation between the Lévy measure $\nu$ of a Lévy process $L$ and existence of sup-variations of the process. 
The Blumenthal-Getoor index of a Lévy process is defined by

$$
\beta=\inf \left\{r>0: \int_{[-1,1]}|x|^{r} \nu(\mathrm{d} x)<\infty\right\} .
$$

If $\beta<r$ then pathwise (Lépingle (1976), Hudson and Mason (1976))

$$
\left[L_{\delta}\right]^{[r]}(t) \rightarrow \sum_{0<s \leq t}|\Delta L(s)|^{r}<\infty
$$

whereas in general $\left[L_{\delta}\right]^{[r]}(t) \rightarrow \infty$ when $r \leq \beta$.

Furthermore (see Sato (1999, Theorem 21.9)), with $r=1$ we have $[L]^{[1]}<\infty$ or $=\infty$ according as $\beta \leq 1$ or $1<\beta(<2)$.

Some extensions to additive processes are considered in Woerner (2002).

Power variation and semimartingales Let $X$ be a semimartingale. Lépingle (1976) considered sup-variations of semimartingales generally and showed that $[X]^{[r]}(t)<\infty$ for every $r>2$ while for $1<r<2$ we have

$$
\left[X_{\delta}\right]^{[r]}(t) \rightarrow \sum_{0<s \leq t}|\Delta X(s)|^{r}
$$

provided $\langle X\rangle_{t}=0$ and

$$
\sum_{0<s \leq t}|\Delta X(s)|^{r}<\infty
$$

Sup-r-variation and integration We briefly recall the role of sup-variation in the theory of integration.

Young (1936) extended the Stieltjes integral to allow for integration in cases where the integrand and/or the integrator may be of unbounded variation. Dudley (1992) and Dudley and Norvaiša (1999) extended the concept further, and Mikosch and Norvaiša (2000) applies the theory to give path-by-path solutions to many basic stochastic integral equations. The main condition for the existence of such solutions is that $0<r<2$.

An annotated bibliography on power variation is available in Dudley, Norvaiša, and Jinghua Qian (1999). See also Dudley and Norvaiša (1998). We also refer to the related work of Lyons on rough paths, see Lyons (1994) and Bass, Hambly, and Lyons (2002) and references given there.

\section{References}

Andersen, T. G., T. Bollerslev, and F. X. Diebold (2004). Parametric and nonparametric measurement of volatility. In Y. Ait-Sahalia and L. P. Hansen (Eds.), Handbook of Financial Econometrics. Amsterdam: North Holland. Forthcoming.

Barndorff-Nielsen, O. E. (2001). Superposition of Ornstein-Uhlenbeck type processes. Theory of Probability and its Applications 45, 175-194.

Barndorff-Nielsen, O. E., S. E. Graversen, J. Jacod, M. Podolsky, and N. Shephard (2004). Bipower variation. Unpublished paper: Nuffield College, Oxford.

Barndorff-Nielsen, O. E., E. Nicolato, and N. Shephard (2002). Some recent developments in stochastic volatility modelling. Quantitative Finance 2, 11-23.

Barndorff-Nielsen, O. E. and N. Shephard (2001a). Modelling by Lévy processes for financial econometrics. In O. E. Barndorff-Nielsen, T. Mikosch, and S. Resnick (Eds.), Lévy Processes - Theory and Applications, pp. 283-318. Boston: Birkhäuser. 
Barndorff-Nielsen, O. E. and N. Shephard (2001b). Non-Gaussian Ornstein-Uhlenbeck-based models and some of their uses in financial economics (with discussion). Journal of the Royal Statistical Society, Series B 63, 167-241.

Barndorff-Nielsen, O. E. and N. Shephard (2002). Econometric analysis of realised volatility and its use in estimating stochastic volatility models. Journal of the Royal Statistical Society, Series B 64, 253-280.

Barndorff-Nielsen, O. E. and N. Shephard (2003). Realised power variation and stochastic volatility. Bernoulli 9, 243-265 and 1109-1111.

Barndorff-Nielsen, O. E. and N. Shephard (2004). Econometric analysis of realised covariation: high frequency covariance, regression and correlation in financial economics. Econometrica 72, 885-925.

Bass, R., B. Hambly, and T. Lyons (2002). Extending the Wong-Zakai theorem to reversible Markov processes. Journal of the European Mathematical Society 4, 237-269.

Dudley, R. (1992). Fréchet differentiability, p-variation and uniform Donsker classes. Annals of Probability 20, 1968-1982.

Dudley, R. and R. Norvaiša (1998). An introduction to $p$-variation and Young integrals; with emphasis on sample functions of stochastic processes. MaPhySto Lecture Notes 1998-1, Aarhus University: MaPhySto.

Dudley, R. and R. Norvaiša (1999). Product integrals, Young integrals and $p$-variation. In R. Dudley and R. Norvaiša (Eds.), Differentiability of Six Operators on Nonsmooth Functions and p-variation. New York: Springer-Verlag. Lecture Notes in Mathematics 1703.

Dudley, R., R. Norvaiša, and Jinghua Qian (1999). Bibliographies on $p$-variations and $\phi$ variation. In R. Dudley and R. Norvaiša (Eds.), Differentiability of Six Operators on Nonsmooth Functions and p-variation. New York: Springer-Verlag. Lecture Notes in Mathematics 1703.

Feller, W. (1971). An Introduction to Probability Theory and Its Applications (2 ed.), Volume 2. New York: John Wiley.

Fristedt, B. and S. Taylor (1973). Strong variation for the sample functions of a stable process. Duke Mathematics Journal 40, 259-278.

Gnedenko, B. W. and A. N. Kolmogorov (1954). Limits Distributions for Sums of Independent Random Variables. Cambridge, Massachusetts: Addison-Wesley.

Hudson, W. N. and J. D. Mason (1976). Variational sums for additive processes. Proceeding of the Americian Mathematical Society 55, 395-399.

Jacod, J. (1994). Limit of random measures associated with the increments of a Brownian semimartingale. Unpublished paper: Laboratorie de Probabilities, Universite $\mathrm{P}$ and $\mathrm{M}$ Curie, Paris.

Jacod, J. and P. Protter (1998). Asymptotic error distributions for the Euler method for stochastic differential equations. Annals of Probability 26, 267-307.

Kallsen, J. and A. N. Shiryaev (2002). Time change representation of stochastic integrals. Theory of Probability and its Applications 46, 522-528.

Lépingle, D. (1976). La variation d'ordre p des semi-martingales. Zeitschrift fur Wahrscheinlichkeitstheorie und verwandte Gebiete 36, 295-316.

Loève, M. (1977). Probability Theory I (4 ed.). New York: Springer.

Lyons, T. (1994). Differential equations driven by rough signals. I. An extension of an inequality by L.C.Young. Mathematical Research Letters 1, 451-464. 
Mikosch, T. and R. Norvaiša (2000). Stochastic integral equations without probability. Bernoulli 6, 401-434.

Mykland, P. and L. Zhang (2005). ANOVA for diffusions. Annals of Statistics 33. Forthcoming.

Sato, K. (1999). Lévy Processes and Infinitely Divisible Distributions. Cambridge: Cambridge University Press.

Shiryaev, A. N. (1999). Essentials of Stochastic Finance: Facts, Models and Theory. Singapore: World Scientific.

Woerner, J. (2002). Variational sums and power variation: a unifying approach to model selection and estimation in semimartingale models. Statistics and Decisions 21, 47-68.

Young, L. C. (1936). An inequality of the Hölder type, connected with Stieltjes integration. Acta Mathematica 67, 251-282. 